Mots. Les langages du politique

$70 \mid 2002$

La politique en chansons

\title{
Revendication et politiques en paroles : chansons de la communauté noire américaine
}

Claims and Policies in the form of Lyrics: Black-Americains songs

Reivindicación y política en la letra : canciones de la comunidad afroamericana

\section{Valérie Bonnet}

\section{(2) OpenEdition}

Journals

Édition électronique

URL : https://journals.openedition.org/mots/9383

DOI : $10.4000 /$ mots.9383

ISSN : 1960-6001

Éditeur

ENS Éditions

Édition imprimée

Date de publication : 1 novembre 2002

Pagination : 65-78

ISBN : 2-84788-016-X

ISSN : 0243-6450

\section{Référence électronique}

Valérie Bonnet, «Revendication et politiques en paroles : chansons de la communauté noire

américaine », Mots. Les langages du politique [En ligne], 70 | 2002, mis en ligne le 07 mai 2008, consulté le 22 avril 2022. URL : http://journals.openedition.org/mots/9383 ; DOI : https://doi.org/10.4000/ mots.9383 


\section{Revendication et politiques en paroles : chansons de la communauté noire américaine}

Notre propos n'est pas de retracer l'histoire de la communauté noire américaine, ni même celle de sa lutte, longue et complexe, mais de placer les jalons d'un discours politique qui transparait dans les chansons populaires. C'est donc l'évolution parallèle de la musique populaire noire américaine, et du discours militant par les moyens de sa mise en œuvre que nous allons tenter de décrire ici.

La musique est un mode d'expression de la communauté noire que les blancs essaient de contrôler depuis l'importation des premiers esclaves, craignant, à juste titre comme le démontrera l'histoire, qu'il ne les incite à la révolte ${ }^{1}$. Les esclaves se voient interdire toute pratique instrumentale, entre autres procédés d'acculturation que subit la communauté. Cependant, le flot continu de la traite jusqu'en 1859 leur permet de garder une forme de contact culturel avec leur continent d'origine et de maintenir une mémoire qui passe, bien entendu, par la musique.

Seules les worksongs, en ce qu'elles favorisent la rentabilité des travailleurs, sont autorisées. Mais là encore, des limites sont fixées : elles ne doivent être ni mélancoliques, ni nostalgiques. Comme le soulignent Philippe Carles et Jean-Louis Comolli, la musique noire est systématiquement « détourné[e] vers l'entertainment (divertissement, passetemps)» (P. Carles \& J.-L. Comolli, 2000, p. 199). Le blues, avec son rôle cathartique - le blues étant « la musique des « idées noires » de l'attendrissement amer» selon le Dictionnaire Historique de la Langue Française (article blues) - se joue donc dans la clandestinité. Cette musique Lyon.

- Groupe Rhéma, EA 1996 Langues, Textes, Images, 65 avenue du Doyenné, 69005

1. Voir P. Carles et J.-L. Comolli, 2000, Free jazz Black power, Folio, 438 p. 
constitue donc une forme de subversion, d'autant plus qu'elle aborde des thèmes interdits comme l'argent ou le sexe.

Les chants religieux (spirituals, gospel songs) sont également étroitement associés à la politique et à la revendication sociale. En effet, si dans un premier temps, les blancs surveillaient les églises afro-américaines, elles furent progressivement abandonnées aux pasteurs noirs, qui constituèrent en quelque sorte les premiers leaders politiques de communauté. Les textes sacrés y sont réinterprétés à travers le filtre de l'esclavage, et nul besoin est d'expliciter quelle lecture y était faite du séjour des Hébreux en Égypte. La subversion de ces chants religieux, interprétés ouvertement, repose sur l'instauration d'un double langage qui permet aux esclaves de communiquer à l'insu de leurs maitres.

On le constate, dès ses premiers pas, la musique est l'un des nombreux terrains sur lequel se joue la domination d'une communauté sur une autre. D'autant plus subversive qu'elle est dument contrôlée, elle oscille entre une fonction cathartique clandestine et la transmission de messages d'espoir de jours meilleurs sous couvert d'amusement. C'est la voie même que lui avait imposée l'homme blanc pour mieux la neutraliser, celle de l'entertainment - dans les faits, le blues ne sera jamais une musique militante $^{2}$ - qui en fera le médium le plus puissant dans la transmission des messages de révolte.

\section{La soul : le topos amoureux}

La musique noire était depuis le milieu des années 1950 une musique populaire de large diffusion dont le but, contrairement au blues, était de divertir les masses. Cette musique pour teenagers traitait essentiellement $\mathrm{du}$ thème de l'amour. Certes, ce thème était également présent dans le blues, mais la soul l'aborde sous des aspects positifs, vantant l'amour heureux. En cela, elle est une musique d'espoir, qui remplit également une fonction cathartique, mais selon un fonctionnement inverse de celui du blues.

Cet espoir est surtout marqué par une confiance en un futur meilleur, les épreuves évoquées ne pouvant qu'être surmontées. Résolument optimistes, les paroles de la soul music n'en sont pas pour autant irréalistes : tout comme dans le blues, la situation est décrite telle qu'elle est. Mais,

2. Voir M. Haralambos, 1974, Soul Music, The Birth Of A Sound In Black America, New-York, Da Capo Press, p. 53-92. 
alors que, dans le blues, la situation future ne peut être que la continuation du présent, dans la soul, il ne fait pas de doute qu'il est possible d'en venir à bout.

On conçoit dès lors ce que l'optimisme de ce discours peut avoir de décisif pour le point qui nous occupe : si la soul pose les difficultés relationnelles comme une simple étape vers un futur meilleur, elle ne peut que porter un message d'espoir pour la situation de la communauté noire. Ainsi, les textes des chansons ne tardent pas à être réinterprétés dans un contexte social. Le titre We Gonna Make It (Nous réussirons) de Little Milton (1965), qui expose les difficultés inhérentes à la construction d'un couple, est de ceux-ci.

C'est aussi le cas d'un titre d'Otis Redding intitulé Respect (1965), et qui traite également des relations de couple. Perçu comme tel au moment de sa sortie, il sera réenregistré en 1967 par Aretha Franklin, qui replace les paroles d'un point de vue féminin. La situation a changé en deux ans, et le texte est interprété comme une chanson politique, alors que la chanteuse elle-même s'en défend. De même, le Dancing In the Street (Danser dans la rue) de Martha Reeves and the Vandellas (1964) sert d'hymne aux émeutiers de Detroit, en 1967. Tell It Like It Is (Dis-les choses telles qu'elles sont), titre d'une chanson d'amour d'Aaron Neville (1966) deviendra même l'expression consacrée utilisée par les activistes noirs américains pour réclamer des autorités un discours transparent.

Que l'on traduise ces chansons ainsi, alors que leurs interprètes euxmêmes nient qu'elles aient de telles implications, indique à quel point les noirs américains recherchent des porte-paroles susceptibles d'exprimer leur colère et leurs espoirs. Mais c'est également la résultante d'une longue tradition de double entente, qui prend ses racines dans les chants religieux évoqués précédemment, et que la situation de classe dominée, qui survit souvent au moyen d'expédients, a favorisée.

Cependant, le mouvement noir ne va pas tarder à changer d'orientation, et le pacifisme teinté de religiosité de Martin Luther King sera supplanté par le marxisme des Black Panthers. Au discours optimiste de la première partie des années 1960 - le mouvement pour les droits civiques est porteur d'espoir - succède l'impatience d'une communauté qui ne supporte plus d'attendre l'égalité des droits. L'assassinat de Malcom X, le 21 février 1965 , ne fera qu'accentuer ce sentiment. Les partis qui apparaissent alors sont beaucoup plus radicaux : le Black Panther Party for Self-Defense, le Revolutionary Action Movement, qui prônent tous deux l'action directe, et tournent le dos à l'intégrationnisme militant du pasteur King.

C'est donc en toute logique que la transmission des messages par le 
procédé de la double entente ne peut satisfaire le public comme les artistes $^{3}$. Tout comme l'afro-américain ne supporte plus sa non-existence en tant que citoyen, il ne souffre plus la clandestinité de son message. Bien plus, le mode d'action de la communauté noire est remis en question dans certaines chansons : Nina Simone prophétisera « Do things gradually, and bring more tragedy » (Agissez graduellement et vous provoquerez d'autres tragédies) dans Mississippi Gottham (1964). Le mode de communication issu de la pratique religieuse est remplacé par un discours à caractère politique. Ainsi Curtis Mayfield, après avoir chanté au sein des Impressions des titres dans la droite ligne du gospel, écrira des textes de plus en plus explicites: We're A Winner (Nous sommes des battants, 1967), We're Rolling On (Nous continuons de progresser, 1968), Move On Up (Continue de t'élever, 1968), People Get Up (Peuple, lève-toi, 1969).

\section{L'affirmation d'un discours social}

L'action légale a fait son temps et des émeutes débutent à Harlem en 1964. En aout 1965, elles embrasent le quartier angelino de Watts, et atteignent leur apogée à Detroit et Newmark en 1967. Un pas est désormais franchi: les émeutiers réclament une égalité sociale et économique. Conséquence de la théorisation du discours militant effectuée par Malcom $\mathrm{X}$ - celui-ci dénonce le conditionnement idéologique des noirs par les blancs -, les artistes chantent leur fierté d'être noirs et incitent leur public à se revendiquer comme tel. Engagement et soul music sont désormais associés : des citations des morceaux des Impressions ou de James Brown comme « Say it loud, I'm black and I'm proud» (Proclame-le : je suis noir, et fier de l'être) et « I'd rather die on my feet than keep living on my knees » (Je préfère mourir debout que de continuer à vivre à genoux) sont graphités sur les murs des ghettos ou imprimés sur des t-shirts. Le D. J. Job Cobb souligne le rôle de certaines chansons de soul dans le mouvement noir :

We're Rolling On and songs like that gave a lot of people, and even a lot of civil rights organisations, hope and great strength, and made people believe into it, because actually within the record itself, it as telling you like what to

3. Comme l'indique ce témoignage de l'un des membres des Last Poets : «I began to feel that something more than «I love you » needed to be said » (J'ai senti qu'il était nécessaire d'en dire plus que « je t'aime »). 
expect, and what had happened thus far, so like hold your head up high and keep on going, your day will come (Haralambos, 1974, p. 125) ${ }^{4}$.

Les textes reprennent alors les grandes lignes du discours politique afin d'inciter les membres de la communauté noire à entrer en résistance. $\mathrm{Ce}$ discours, avant tout social, ne constitue pas à proprement parler une transcription explicite des textes du Black Power mais, par certaines caractéristiques linguistiques comme par leur mode de transmission, contribuent à diffuser le message du discours militant auprès des masses. Au nombre des artefacts utilisés, il est des images relativement explicites comme celle de l'oiseau en cage chantée par Nina Simone dans I Wish I Knew How It Would Feel To Be Free (J'aimerais savoir quelle impression cela fait d'être libre, 1967), mais aussi l'utilisation de la phraséologie et de la prononciation du ghetto, à usage exclusivement intra-communautaire ${ }^{5}$.

Ce dernier point est un mode d'affirmation de l'identité noire, qui fait écho à la volonté de construire une communauté afro-américaine. Son accès à la visibilité est à l'opposé de toutes les théories intégrationnistes et constitue un signe de l'abandon de la culpabilité du noir, inculquée par des décennies d'acculturation.

\section{L'affirmation de l'identité}

Les Black Muslims, inspirés du Garveyisme ${ }^{6}$, Malcolm X, qui place la civilisation africaine comme valeur de référence et Rap Brown ${ }^{7}$, qui insiste également sur la nécessité d'assumer son identité noire, feront émerger la notion de fierté noire. À partir de 1968, une thématique nouvelle apparait dans les textes de soul music: la fierté noire, concomitante du mouvement Black is beautiful. Désormais, le noir assume son statut : Young, Gifted and Black (Jeune, douée et noire), affirmera Nina Simone

4. "We're Rolling On [morceau des Impressions] et d'autres chansons de ce type donnèrent de l'espoir et une grande force à beaucoup de gens, et même à beaucoup d'organisations qui luttaient pour les droits civiques, et contribuèrent à ce que les gens se mirent à croire en la cause, parce que c'était comme si les paroles te disaient en quoi espérer et ce qui était arrivé jusqu'à présent, comme si elles te disaient « garde la tête haute et continue, ton jour viendra ».

5. Cette utilisation du dialecte du ghetto est en partie à l'origine du succès de la soul music. En effet, le public y voit une marque de respect de la part des artistes.

6. Marcus Garvey est l'un des premiers leaders noirs, et surtout l'un des premiers porte-paroles de la négritude.

7. Président du Students Non Violent Coordinating Comittee. 
en 1969, Say It Loud, I'm Black And I'm Proud chantera James Brown en 1968. Bien sûr, les paroles d'affirmation d'une fierté noire ne peuvent être détachées d'un contexte politique :

But then out of nowhere I found my pride, And I found that a man don't have to hide (Mais, alors, de nulle part, j'ai trouvé une fierté, et j'ai découvert qu'un homme n'a pas à se cacher, Ghetto Man, Tony Clarke, 1969).

But oh now I've got my pride, deep down inside, And no one will ever take it again (Mais, maintenant, j'ai ma fierté, au plus profond de moi, et personne ne me la reprendra, Time Has Brought About A Change, Willie Hightower, 1970)

Le D. J. Jay Butler soulignera l'évolution dans les mentalités en quelques 14 ans :

Back in 1954, the black community didn't want to be associated with blues. This was because the civil rights bill had passed and at that time more blacks were wearing processes and using Ultra Skin Tone Cream to try and lighten their complexion, because they wanted to be accepted by whites. But it's since changed. The black community wants to be accepted as black Americans. I don't wanna be a white American, I wanna be a black American. It's like the Detroit Emeralds'song (I'm An Ordinary Man / Take Me The Way I Am). There's a two fold meaning in all these soul songs. Take Salomon Burke's Take Me As I Am. [...] Back in 1950s we were trying to be accepted by white Americans on their terms. Now accept me as I am, accept my nappy hair, accept me period (Haralambos, 1974, p. 146) ${ }^{8}$.

Le chanteur de soul prend alors une autre importance. Si le public pouvait (en cachette) se projeter dans les malheurs évoqués par le chanteur de blues, il en est de même avec les artistes de soul music, comme le montre cette assertion du D. J. Lee Garrett :

Soul singers are important because it's someone who is in the light, the public light, someone the public looks up to has touched on their problem, and it

8. «En 1954, la communauté noire ne voulait pas être associée au blues. Ceci en raison du fait que le projet de loi sur les droits civiques avait été voté et à cette époque, plus de noirs se transformaient (pour ressembler à des blancs) et utilisaient de la Ultra Skin Tone Cream pour éclaircir leur teint, parce qu'ils voulaient être acceptés par les blancs. Mais cela a changé depuis. La communauté noire veut être acceptée comme des noirs américains. Je ne veux pas être un américain blanc, je veux être un noir américain. C'est comme la chanson de Detroit Emerald (Je suis un homme ordinaire, prenez-moi comme je suis). Il y a un double sens dans toutes ces chansons soul. Prenez la chanson de Salomon Burke, Prendsmoi comme je suis [...]. Dans les années 1950, nous essayions d'être acceptés par les Américains blancs selon leurs conditions. Maintenant, c'est «accepte-moi comme je suis, accepte mes cheveux crépus. Un point, c'est tout » (Haralambos, 1974, p. 146). 
makes you smile to know that someone understands yours problems (Haralambos, 1974, p. 107) ${ }^{9}$.

Le chanteur de soul prend alors valeur d'exemple aux yeux de ses congénères. À tel point que c'est à James Brown que les maires de Boston et Washington font appel pour tenter de calmer les émeutes qui suivirent l'assassinat de Martin Luther King en avril 1968. Les D. J. contribuent à créer ce statut en insistant sur le message dont sont porteurs les textes, citant les passages les plus importants avant de passer les disques, les répétant et les paraphrasant après que le disque a été diffusé. Bien plus, ils les érigent en vérité - au nombre des antiennes des animateurs radio, il convient de noter des expressions comme « ain't that the truth» (n'est-ce pas la vérité?), " that's the natural truth » (c'est la pure vérité), "You'd better believe it » (vous devriez croire [ce que je vous dis]) et, bien sûr, « tell it like it is ».

Les artistes de soul eux-mêmes prennent en charge ce rôle de propagateur en introduisant leurs chansons par une invite à l'écoute, des expressions comme « listen » (écoute), " hear what I say » (écoute ce que je te dis). Cette pratique qui provient du gospel - beaucoup d'artistes de soul (Aretha Franklin, Curtis Mayfield, James Brown, Sam Cooke, Syl Johnson) ont appris à chanter à l'église ou dans des formations gospel - et avait pour objet d'impliquer le fidèle, trouve dans le combat militant toute sa dimension : l'insistance sur le message délivré et l'invite à une implication de l'auditoire.

\section{La constitution d'une communauté}

L'artiste ne se contente pas d'interpeller son public par des propos introducteurs, ses paroles n'ont pas seulement valeur d'exemple, elles sont également des messages directs d'un noir à un autre noir :

Y'see if you have white light brown skin and high yellow, you're still black, so we got to stick together now (Que tu aies une peau marron clair et des cheveux décolorés, tu es toujours un noir, c'est pourquoi nous devons nous serrer les coudes, Is It Because I'm Black, Syl Johnson, 1968),

You Can Make It If You Try, (Tu peux le faire si tu essayes, Sly Stone, 1968).

9. «Le chanteur de Soul est important parce qu'il est sous le feu des projecteurs, en pleine lumière, c'est quelqu'un que le public respecte pour avoir abordé ses problèmes, et cela vous rend le sourire de savoir que quelqu'un comprend vos problèmes » (Haralambos, 1974, p. 107). 
Cette apostrophe, qui utilise, bien entendu le you - fort pratique en anglais en raison de l'ambigüité entre le singulier et le pluriel - est renforcée par l'utilisation progressive de l'impératif, appel direct à une implication dans l'action de la communauté : Get Up, Get Involved, Get Into It (Lève-toi, implique-toi, entre dans (le mouvement), James Brown, 1968), Move On Up (The Impressions, 1968), Say It Loud, I'm Black And I'm Proud (J. Brown, 1968), Stand! (Résiste!, Sly Stone, 1969). De même, l'ambigüité quant à la personne - singulier ou pluriel - dans l'utilisation de l'impératif contribue à la création d'un esprit communautaire.

Mais surtout, la soul utilise la première personne du pluriel, mettant ainsi en avant l'idée de communauté :

We Are Rolling On (1968),

We're A Winner (1967),

We got talent we can use (Nous avons des talents que nous pouvons utiliser,

I Don't Want Nobody To Give Me Nothing, J. Brown, 1969).

Ce changement est loin d'être anodin, et marque un pas par rapport au blues. Le blues était une musique de l'individu. Il avait certes une valeur d'exemple, car la condition du chanteur de blues était celle de son public, mais ne transcrivait en aucune façon l'idée de communauté. Le chanteur de blues utilisait le $I$, et ne parlait que de lui. À cet aspect sémantique faisaient écho les performances scéniques : quand il interpellait le public, la réponse de celui-ci était individuelle. Inversement, les chanteurs de soul apostrophent un public qui répond à l'unisson.

En 1967, parait le manifeste du Black Power, qui revendique le contrôle des institutions économiques, politiques et sociales; il est désormais temps de passer à l'action. Et, de fait, les mots d'ordre politiques de plus en plus explicites apparaissent dans les chansons : "But I say we won't quit moving, Until we get what we deserve, [...] We'd rather die on our feet than keep living on our knees » (Nous ne cesserons d'agir jusqu'à que nous obtenions ce que nous méritons. [...] Nous préférons mourir debout plutôt que continuer à vivre à genoux) (Say It Loud, I'm Black And I'm Proud, J. Brown, 1968). Pour preuve du poids de ce type de déclaration, cette phrase coutera à James Brown une partie du public blanc qu'il était le premier artiste noir a avoir réellement.

En effet, la music soul touche un public extrêmement important, tant par le canal des radios que par les performances scéniques, et c'est cette diffusion de masse qui donne son poids au message qu'elle véhicule. Si les textes de soul présentent ce que ressentent les noirs, ils les incitent également à penser autrement. 


\title{
Le discours politique
}

\begin{abstract}
À partir du début des années 1970, les références politiques sont affichées :
\end{abstract}

Are You Really Ready For Black Power? (Es-tu vraiment prêt pour le pouvoir noir?, Gary Bird, 1970).

When The Revolution Comes (Quand la révolution arrivera, The Last Poets, 1970),

Brothers hold your hands across the nation, Wanna gather now than ever before, Freedom right now or else damnation (Frères, tendez-vous la main à travers le pays, maintenant nous allons nous unir comme nous ne l'avons jamais fait, (nous voulons) la liberté immédiate ou bien la damnation), Stand Up And Be Counted, The Flames, 1971.

Ever since they passed the civil rights, Those fires have been lighting up the nights, And they say they ain't gonna stop, «til we all have equal rights » (Dem Niggers Ain 't playing, The Watts Prophets, 1971) (Depuis qu'ils ont voté les droits civiques, ces feux ont éclairé les nuits, et ils ont dit qu'ils n'arrêteraient pas, « jusqu'à que nous ayons l'égalité des droits »).

Apparaissent alors des formations comme les Last Poets, ou les Watts Prophets qui ne se destinent en aucune manière au divertissement. Leur but est de diffuser un discours politique, ce qui ne les empêche pas de remporter des succès commerciaux : le premier album des Last Poets s'est vendu à 300000 exemplaires simplement par le bouche à oreille, atteignant ainsi la $40^{\mathrm{e}}$ place des charts américains. Leur poids dans le contexte politique n'est pas davantage à remettre en question, puisque le FBI place les Watts Prophets sur la Unamerican Activities List pour 7 ans, restreignant ainsi leur sphère d'action, ceux-ci ne pouvant plus se produire en concert dans les universités.

La musique est désormais le médium privilégié de la communauté afro-américaine, à tel point que les artistes sont courtisés par les membres du Black Panther Party : ce sera le cas de Jimi Hendrix, qui présente cet intérêt de toucher le public blanc.

C'est également par ce médium que la communauté noire règle ses comptes et laisse apparaitre toutes les dissensions internes du mouvement : Gil Scott-Heron critique les politiciens noirs dans The Revolution Will Not Be Televised (La révolution ne sera pas diffusée à la télévision, 1971), les Last Poets et Gary Bird stigmatisent le manque d'investissement et de conscience politique de certains membres de la communauté 
(Niggers Are Scared Of Revolution, Last Poets, 1971; Are You Really Ready For Black Power? Gary Bird, 1970) ${ }^{10}$.

En effet, la révolution du Black Power est une révolution marxiste articulation entre les données raciales, juridiques, sociales, culturelles et politiques qui avaient été mise en place par Malcolm X et qui sera reprise par le Black Panther Party -, et implique tout refus de compromission avec le système capitaliste :

The idea seems to be that money will make us free,

Black capitalism says the system is what we really need,

But what difference does it make in the colour of that hand,

If it takes food from the mouths of black people in order to become a richer man?

(Are You Really Ready For Black Power? Gary Bird, 1970) ${ }^{11}$.

Ainsi, le morceau de James Brown, I Don't Want Nobody To Give Me Nothing (Je n'ai besoin de personne pour me donner quoi que ce soit), sera-t-il sévèrement critiqué par les activistes noirs, dans la mesure où il affiche sa croyance dans le capitalisme noir : "When some of us make money, We just care about our people » (Quand certains d'entre nous gagnent de l'argent, ils prennent simplement soin des nôtres).

D'autre part, sont montrés du doigt les noirs qui ne veulent changer la situation que pour prendre la place des blancs et agir comme eux :

Niggers come in from work and change into pimping clothes

And hit the street to make some quick change

Niggers change their hair from black to red to blond

And hope like hell their looks will change

(Niggers Are Scared Of Revolution, Last Poets, 1971) ${ }^{12}$.

Face à un front révolutionnaire qui se délite (le Black Panther Party souffre de nombreuses dissensions et sa culture révolutionnaire l'a coupé

10. Respectivement Les nègres ont peur de la révolution et Es-tu vraiment prêt pour le pouvoir noir?

11. «Il semblerait que l'argent nous rende libre. Le capitalisme noir dit que le système est ce dont nous avons réellement besoin. Mais la couleur de la main qui prend la nourriture de la bouche des noirs pour devenir plus riche a-t-elle une importance? »

(Are You Really Ready For Black Power?, Gary Bird, 1970)

12. «Les nègres rentrent du travail et mettent leur vêtements de maquereaux, et arpentent les rues pour se faire de l'argent facile. Les nègres teignent leurs cheveux de noir en roux, et de roux en blond et espèrent désespérément que leur apparence va changer » (Niggers Are Scared Of Revolution, Last Poets, 1971). 
de sa base), une petite bourgeoisie noire qui tente de tirer son épingle du jeu, et une situation économique qui n'a pas évolué, la communauté noire ressent un profond sentiment de désillusion. À partir de cette période, beaucoup de chansons évoquent avec désespoir la situation des noirs : Inner City Blues (Le blues du centre urbain, Marvin Gaye, 1971), Sleepin' into darkness (Dormir dans les ténèbres, War, 1971), Hell (Enfer, James Brown, 1974), et l'ironique There's No Place Like America Today, (Il n'y a pas mieux que l'Amérique d'aujourd'hui, Curtis Mayfield, 1975).

C'est là qu'apparait le clivage entre des formations comme les Watts Prophets ou autres Last Poets, ou encore des poètes engagés comme Gil Scott-Heron qui sont avant tout des hérauts politiques, et des artistes comme Marvin Gaye ou James Brown. Les premiers sont les porteparoles d'un discours politique, les autres ceux du peuple. Le titre de ChiLites, For God's Sake Give More Power To The People (1971), est, à ce titre, emblématique de la période : l'impérieux « Give More Power To The People » (Donnez plus de pouvoir au peuple), est étrangement neutralisé par le désespéré « For God's Sake » (Pour l'amour de Dieu).

\section{Après la bataille : l'effet de citation}

Le début des années 1970 annonce l'heure du bilan, alors que sonne le glas de la révolution noire : Mickael James Kirkland constate dans Hang On In There (1972) «I've got too many friends that have shed their blood » (Trop de mes amis ont répandu leur sang); Eddie Kendricks soulignera «I realise how hard you tried, I can see your struggle in your eyes » (Je me suis rendu compte à quel point vous vous êtes battus, je peux voir votre lutte dans vos yeux, My People... Hold On, 1972); "You've killed all our leaders, I don't even have to do nothing to you, You'll cause your own country to fall » (Vous avez tué tous nos leaders, je n'ai rien à faire pour vous, vous causerez la chute de votre propre pays) accusera Stevie Wonder (Big Brother, 1972). Quant à Esther Marrow, elle se contentera de constater Things Ain't Right (Ce n'est pas juste, 1972).

On le sent, la tonalité n'est plus celle des titres chargés d'espoir de cinq ans auparavant, comme le montre l'évolution du titre de Sam Cooke, $-A$ Change Is Gonna Come (Les choses vont changer) - si porteur d'espoir en 1964. En 1970, Willie Hightower chantera Time Has Brought About A Change (Le temps a apporté un changement, 1970). Quatre ans plus tard, le ton a profondément changé et The Alexander Review prophétisera A Change Had Better Come (Les choses feraient mieux de changer, 1974) d'un ton amer. 
La reprise des morceaux d'un autre artiste était une pratique courante dans l'industrie du disque - c'est le cas du RESPECT d'Otis Redding repris par Aretha Franklin -, mais, au fil des années émerge un phénomène d'intertextualité. Les formules les plus marquantes des chansons, qui servent de slogans à la cause noire, sont reprises par d'autres chanteurs :

- Curtis Mayfield cite James Brown : «Now I'm going to say it loud, I'm just as proud as the brothers too " (Maintenant, je vais le proclamer, je suis tout aussi fier que mes frères) (Mighty Mighty Spades and Whitey, The Impressions, 1969);

- tout comme les Watts Prophets : «I go into my act good and loud, say brother, I'm black and I'm proud » (Je passe à l'action ostensiblement; dis-le, frère : je suis noir et fier de l'être), Rappin'Black In A White World, Watts Prophets, 1971).

À partir de la seconde moitié des années 1970, la musique noire engagée entamera une traversée du désert : les artistes se tournent vers l'amusement et la danse.

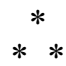

Nous soulignerons pour terminer l'étroite association de la soul music au mouvement pour les droits civiques :

The evolution of soul music closely parallels the stages in the black movement for civil rights and self determination. The scattered soul records from 1954 to 1960 correspond to the black struggle during those years which was intermittent and sporadic and never reached the proportions of a mass movement. The convergence of styles toward soul music from 1960 to 1964 corresponds to the protest movements of the early ' 60 s when the civil rights struggle became a mass movement. The establishment of soul music as the dominant style of black musical expression in the mid-' 60 s correlates with the rise and development of the Black Power Movement (Haralambos, 1974, p. 154) ${ }^{13}$.

13. «L'évolution de la soul musique suit étroitement les étapes du mouvement noir. Les quelques disques de soul de 1954 à 1960 correspondent au combat des noirs durant ces années-là, qui était intermittent et sporadique et n'atteignit jamais les proportions d'un mouvement de masse. La convergence des styles dans la direction de la soul musique de 1960 à 1964 correspond au mouvement de protestation du début des années 1960 quand le combat pour les droits civiques commença à devenir un mouvement de masse. L'établissement de la soul musique comme le genre dominant d'expression musicale noire au milieu des années 1960 a correspondu à l'essor et au développement du Black Power Movement » (Haralambos, 1974, p. 154). 
Symbole de cette association, le concert qui clôturera le Watts Summer Festival de 1972, festival commémorant chaque année les émeutes de Watts. Point d'orgue du festival, il proposera les artistes du label Stax les plus connus du moment. C'est à cette occasion que Jesse Jackson parlera de « liberation through music and lyrics » (libération à travers la musique et les paroles des chansons). Ironie du sort ou refus de regarder la réalité en face, la Black Revolution est moribonde et les paroles du révérend semblent plus conclusives que prophétiques.

Car, en quelques dix ans, certains artistes comme James Brown sont devenus des figures du mouvement, tout aussi importants pour celui-ci que les politiciens. Ainsi, si on dédie des chansons aux uns, comme Push On Jesse Jackson (Fonce Jesse Jackson) par les Pace-Setters, on cite les autres : la chanson James Brown ou "I'm the wish that makes Nina Simone wish she knew how it felt to be free » (Je suis l'incarnation des vœux de Nina Simone quand elle disait qu'elle aimerait savoir ce que c'est que d'être libre) par les Last Poets. En ceci, la musique noire reste fidèle à la fonction mémorielle qui a toujours été la sienne depuis l'arrivée des premiers esclaves.

Sa seconde fonction, la fonction communicatrice/mobilisatrice, marquera également son histoire et jouera un rôle non négligeable dans le mouvement noir américain. C'est au fil de l'évolution de la musique engagée que le premier axe glissera insensiblement vers le second. À l'étiolement de la communauté synchronique d'une période militante particulièrement dynamique, succède une communauté diachronique d'actions orphelines d'un réel mouvement de masse.

Enfin, la musique noire gardera le gout pour le jeu sur les mots et la culture de la double entente : le Push on Jesse Jackson (1972) des PaceSetters fait tout autant référence au verbe "pousser, persévérer, foncer » qu'au mouvement fondé par le révérend en 1971 - le People United to Save Humanity - et le titre de Nina Simone Love Me Or Leave Me (Aime-moi ou quitte-moi) est un pied de nez à un slogan anti-noirs qui fleurit dans les années 1960, «love america or leave it » (aime l'Amérique ou quitte-la). 